\title{
Categorical properties of intuitionistic fuzzy groups
}

\author{
P. K. Sharma ${ }^{1}$ and Chandni ${ }^{2}$ \\ ${ }^{1}$ P.G. department of Mathematics, D.A.V. College \\ Jalandhar, Punjab, India \\ e-mail: pksharma@davjalandhar.com \\ ${ }^{2}$ Lovely Professional University \\ Phagwara, India \\ e-mail: chandni16041986@gmail.com
}

Received: 5 June 2021

Accepted: 18 September 2021

\begin{abstract}
The category theory deals with mathematical structures and relationships between them. Categories now appear in most branches of mathematics and in some areas of theoretical computer science and mathematical physics, and acting as a unifying notion. In this paper, we study the relationship between the category of groups and the category of intuitionistic fuzzy groups. We prove that the category of groups is a subcategory of category of intuitionistic fuzzy groups and that it is not an Abelian category. We establish a function $\beta: \operatorname{Hom}(A, B) \rightarrow$ $[0,1] \times[0,1]$ on the set of all intuitionistic fuzzy homomorphisms between intuitionistic fuzzy groups $A$ and $B$ of groups $G$ and $H$, respectively. We prove that $\beta$ is a covariant functor from the category of groups to the category of intuitionistic fuzzy groups. Further, we show that the category of intuitionistic fuzzy groups is a top category by establishing a contravariant functor from the category of intuitionistic fuzzy groups to the lattices of all intuitionistic fuzzy groups.
\end{abstract}

Keywords: Intuitionistic fuzzy group, Intuitionistic fuzzy homomorphism, Category, Covariant functor, Contravariant functor.

2020 Mathematics Subject Classification: 03E72, 08A72.

\section{Introduction}

Category theory is the study of mathematical structures by means of the relationships between them. It provides a framework for considering the diverse contents of mathematics from the 
logical to the topological. Category theory was developed by Saunders Mac Lane and Samuel Eilenberg in [6]. Initially, it was associated with algebraic topology and geometry. In recent years category theory has been associated with areas as diverse as computability, algebra and quantum mechanics. For conceptual concepts about Category theory and related areas, We refer to follows Tom Leinster [9], Steve Awodey [3], O. Wyler [13] and Kim, Lim, Lee, Hur [8].

The theory of Intuitionistic Fuzzy Sets introduced by K. T. Atanassov [1,2] plays an important role in modern mathematics. It is a generalization to the theory of Fuzzy Sets given by L. A. Zadeh [15]. R. Biswas was the first one to apply the theory of intuitionistic fuzzy sets (IF sets) in Algebra and introduced the notion of intuitionistic fuzzy subgroup of a group in [4]. After this many mathematicians have worked on it and study intuitionistic fuzzy group (IFG) in different contexts for example: Zhan and Tau [16] studied IFGs with operators, Chuangu [5] studied IFGs with the operation on the elements based on the IF relations. Fathi and Salleh [7] studied IFGs based on the notion of IF space. Yuan and Xing [14] studied IFGs based on the definition of neighbourhood relation between an element and the IF Sets. Sharma [10] studied IFG based on $(\alpha, \beta)$-cut sets of IFSs. The notion of IF Abelian groups are also defined and studied by Sharma in [11]. He also studied the representation of IF groups in [12]. The aim of this paper is to bring the study of intuitionistic fuzzy groups into a categorical perspective in order to set the ground for future work.

It can be easily seen that the classes of all intuitionistic fuzzy groups and intuitionistic fuzzy homomorphisms constitute the objects and morphisms, respectively, of the category $\mathbf{C}_{\text {IFG }}$ where composition of morphisms is the usual composition of functions. Furthermore, for any two intuitionistic fuzzy groups $A$ and $B$, the set $\operatorname{Hom}(A, B)$ of all intuitionistic fuzzy homomorphisms forms an Abelian group under the usual addition of intuitionistic fuzzy homomorphisms. In Section 3, we establish a covariant functor from the category of groups $\mathbf{C}_{\mathbf{G}}$ to the category of intuitionistic fuzzy groups $\mathbf{C}_{\mathbf{I F G}}$. We show that $\mathbf{C}_{\mathbf{I F G}}$ is indeed an additive category although it is never an Abelian category (Section 4).

In the process, we introduce an important technical tool to "optimally intuitionistically fuzzify" families of homomorphisms. This ability to intuitionistic fuzzify provides $\mathbf{C}_{\text {IFG }}$ with the structure of a top category over $\mathbf{C}_{\mathbf{G}}$ (Section 4$)$.

\section{Preliminaries}

As was explained in the Introduction, we shall analyse the category of intuitionistic fuzzy groups using known notions from Category theory. Thus, it is important to recall the notions that are necessary to define categories and functors and also some related results on intuitionistic fuzzy groups and intuitionistic fuzzy homomorphisms.

Definition 1 ([3]). A category $C$ is a quadruple $(O b$, Hom, $i d, \circ)$ consisting of:

(Cl) Ob, an object class;

(C2) $\operatorname{Hom}(X, Y)$ a set of morphisms is associated with each ordered object pair $(X, Y)$; 
(C3) a morphism $i d_{X} \in \operatorname{Hom}(X, X)$, for each object $X$;

(C4) a composition law holds, i.e., if $f \in \operatorname{Hom}(X, Y)$ and $g \in \operatorname{Hom}(Y, Z), g \circ f \in \operatorname{Hom}(X, Z)$; such that it satisfies following axioms:

(M1) $h \circ(g \circ f)=(h \circ g) \circ f$, for all $f \in \operatorname{Hom}(X, Y), g \in \operatorname{Hom}(Y, Z), h \in \operatorname{Hom}(Z, W)$;

(M2) $i d_{Y} \circ f=f \circ i d_{X}=f, \forall f \in \operatorname{Hom}(X, Y)$;

(M3) a set of $\operatorname{Hom}(X, Y)$ morphisms are pairwise disjoint.

Definition 2 ([13]). The opposite category $C^{o p}$ of a given category $C$ is formed by reversing the arrows, i.e., for each ordered object pair $(X, Y)$

$$
\operatorname{Hom}_{C} \text { op }(Y, X)=\operatorname{Hom}_{C}(X, Y)
$$

Definition 3 ([13]). A category $D$ is a subcategory of the category $C$ if $o b(D) \subseteq O b(C)$, $\operatorname{Hom}_{D}(X, Y) \subseteq \operatorname{Hom}_{C}(X, Y)$ for ordered object pair $(X, Y)$ and composition of morphisms and the identity of $D$ should be the same as that of $C$.

Definition 4 ([13]). For ordered object pair $(X, Y)$ of $D$, a full subcategory of a category $C$ is a category $D$ if $o b(D) \subseteq O b(C)$ and $\operatorname{Hom}_{D}(X, Y)=\operatorname{Hom}_{C}(X, Y)$.

Definition 5 ([13]). A category $C$ is called an Abelian category if and only if it satisfies the following axioms:

1. $C$ is an additive category.

2. Every morphism in $C$ has kernel and a cokernel.

3. Every monomorphism in $C$ is the kernel of its cokernel.

4. Every epimorphism in $C$ is the cokernel of its kernel.

Definition 6 ([13]). The Category $C^{S}$ is said to be a top category over category $C$, if for a given object $A$ in $C, A$ form a complete lattice $s A$ in $C^{S}$ and if $f: A \rightarrow B$ be a morphism in $C$, the inverse image map $f^{*}: s B \rightarrow s A$ that preserve infima and define a contravariant functor.

Proposition 1 ([3]). The collection of all groups and group homomorphisms is a category. This category is denoted by $\boldsymbol{C}_{\boldsymbol{G}}$.

Proof. A category groups $\mathbf{C}_{\mathbf{G}}$ consisting of:

$(\mathrm{Cl})$ a class of objects $=O b\left(\mathbf{C}_{\mathbf{G}}\right)=$ all groups;

(C2) a set of $\operatorname{Hom}(G, H)$ morphisms for each ordered object pair $(G, H)=$ Set of all group homomorphisms;

(C3) for each object $G$ an identity morphism $i d_{G} \in \operatorname{Hom}(G, G)$; 
(C4) Composition law: For each pair of morphisms $f \in \operatorname{Hom}(G, H)$ and $g \in \operatorname{Hom}(H, P)$, a morphism $g \circ f \in \operatorname{Hom}(G, P)$ the following diagram commutes

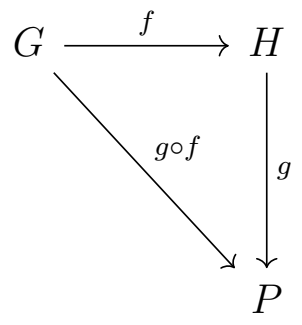

(G1) $h \circ(g \circ f)=(h \circ g) \circ f$, for all $f \in \operatorname{Hom}(G, H), g \in \operatorname{Hom}(H, P)$ and $h \in \operatorname{Hom}(P, Q)$; i.e., the following diagram is commutative

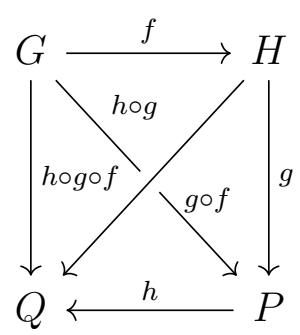

Therefore associativity of the composition holds.

(G2) $i d_{H} \circ f=f \circ i d_{G}=f, \forall f \in \operatorname{Hom}(G, H)$;

(G3) the sets $\operatorname{Hom}(G, H)$ are pairwise disjoint.

Remark 1. A category of groups $\boldsymbol{C}_{\boldsymbol{G}}=\left(O b\left(\boldsymbol{C}_{\boldsymbol{G}}\right), \operatorname{Hom}\left(\boldsymbol{C}_{\boldsymbol{G}}\right), \circ\right)$ consisting of two classes:

(i) a class of objects $=O b\left(\boldsymbol{C}_{\boldsymbol{G}}\right)=$ all groups;

(ii) a class of morphisms $\operatorname{Hom}\left(\boldsymbol{C}_{\boldsymbol{G}}\right)=\bigcup\left\{\operatorname{Hom}_{\boldsymbol{C}_{\boldsymbol{G}}}(G, H): G, H \in O b\left(\boldsymbol{C}_{\boldsymbol{G}}\right)\right\}$, where $\operatorname{Hom}_{\boldsymbol{C}_{\boldsymbol{G}}}(G, H)$ are pairwise disjoint sets for each ordered object pair $(G, H)$.

Definition 7 ([5]). Let $C=(O b(C), \operatorname{Hom}(C), i d, o)$ and $D=(O b(D), \operatorname{Hom}(D), i d, o)$ be two categories and let $F_{1}: O b(C) \rightarrow O b(D)$ and $F_{2}: \operatorname{Hom}(C) \rightarrow \operatorname{Hom}(D)$ be maps. Then the quadraple $F=\left(C, D, F_{1}, F_{2}\right)$ is a covariant functor provided:

(i) $X \in O b(C)$ implies $F_{1}(X) \in O b(D)$;

(ii) $f \in \operatorname{Hom}(X, Y)$ implies $F_{2}(f) \in \operatorname{Hom}\left(F_{1}(X), F_{1}(Y)\right), \forall X, Y \in O b(C)$;

(iii) $F_{2}$ preserves composition, i.e., $F_{2}(g \circ f)=F_{2}(g) \circ F_{2}(f), \forall f \in \operatorname{Hom}(X, Y)$ and $g \in$ $\operatorname{Hom}(Y, Z)$;

(iv) F preserves identities, i.e., $F_{2}\left(e_{X}\right)=e_{F_{1}(X)}, \forall X \in O b(C)$.

Remark 2 ([5]).

(i) Instead of $F_{1}(X)$ we write $F(X)$.

(ii) In preference to $F_{2}(f)$ we write $F(f)$. 
(iii) We call $F: C \rightarrow D$ a functor from $C$ to $D$.

(iv) A functor defined above is called a covariant functor that preserves both:

- The domains, the codomains and identities.

- The composition of arrows, especially it preserves the path of the arrows

(v) A contravariant functor $F$ is similar to the covariant functor in addition to the other side of the arrow, $F(f): F(Y) \rightarrow F(X)$ and $F(g \circ f)=F(f) \circ F(g), \forall f \in \operatorname{Hom}(X, Y), g \in \operatorname{Hom}(Y, Z)$.

Thus a contravariant functor $F: C \rightarrow D$ is the same as a covariant functor $F: C^{o p} \rightarrow D$.

Definition 8 ([6,9]). A mapping $A=\left(\mu_{A}, \nu_{A}\right): X \rightarrow[0,1] \times[0,1]$ is called an intuitionistic fuzzy set on $X$ if $\mu_{A}(x)+\nu_{A}(x) \leq 1$ for all $x \in X$, where the mappings $\mu_{A}: X \rightarrow[0,1]$ and $\nu_{A}$ : $X \rightarrow[0,1]$ denote the degree of membership (namely $\left.\mu_{A}(x)\right)$ and the degree of non-membership (namely $\nu_{A}(x)$ ) of each element $x \in X$ to $A$, respectively.

An intuitionistic fuzzy set $A$ in $X$ can be represented as an object of the form

$$
A=\left\{<x, \mu_{A}(x), \nu_{A}(x)>: x \in X\right\}
$$

where the functions $\mu_{A}: X \rightarrow[0,1]$ and $\nu_{A}: X \rightarrow[0,1]$ denote the degree of membership (namely $\mu_{A}(x)$ ) and the degree of non-membership (namely $\nu_{A}(x)$ ) of each element $x \in X$ to $A$, respectively, and $0 \leq \mu_{A}(x)+\nu_{A}(x) \leq 1$ for each $x \in X$.

Remark 3. (i) When $\mu_{A}(x)+\nu_{A}(x)=1$, i.e., $\nu_{A}(x)=1-\mu_{A}(x)=\mu_{A^{c}}(x)$. Then A is called a fuzzy set.

(ii) We denote the IFS $A=\left\{\left\langle x, \mu_{A}(x), \nu_{A}(x)>: x \in X\right\}\right.$ by $A=\left(\mu_{A}, \nu_{A}\right)$.

Definition 9 ([5,8]). Let $G$ be a group. An IFS $A=\left(\mu_{A}, \nu_{A}\right)$ of $M$ is called an intuitionistic fuzzy subgroup (IFSG)(or intuitionistic fuzzy group) of $G$ if

(i) $\mu_{A}(x+y) \geq \mu_{A}(x) \wedge \mu_{A}(y)$ and $\nu_{A}(x+y) \leq \nu_{A}(x) \vee \nu_{A}(y), \forall x, y \in G$;

(ii) $\mu_{A}(-x) \geq \mu_{A}(x)$ and $\nu_{A}(-x) \leq \nu_{A}(x), \forall x \in G$.

Definition 10 ( $[10,13])$. Let $K$ be a subgroup of a group $G$. Then the intuitionistic fuzzy characteristic function of $K$ is denoted by $\chi_{K}$ defined by $\chi_{K}(x)=\left(\mu_{\chi_{K}}(x), \nu_{\chi_{K}}(x)\right)$, where

$$
\mu_{\chi_{K}}(x)=\left\{\begin{array}{ll}
1, & \text { if } x \in K \\
0, & \text { if } x \notin K
\end{array} ; \quad \nu_{\chi_{K}}(x)=\left\{\begin{array}{ll}
0, & \text { if } x \in K \\
1, & \text { if } x \notin K
\end{array} .\right.\right.
$$

Clearly, $\chi_{K}$ is an IFSG of $G$. The IFSGs $\chi_{\{\theta\}}, \chi_{G}$ are called trivial IFSGs of group $G$, where $\theta$ is the zero element of $G$. Any IFSG of group $G$ other than these is called proper IFSG.

Definition 11 ([14]). If $G, H$ are two groups and $A=\left(\mu_{A}, \nu_{A}\right), B=\left(\mu_{B}, \nu_{B}\right)$ are IFSG of $G$ and $H$, respectively. Let $f: G \rightarrow H$ be a homomorphism. Then the map $\bar{f}: A \rightarrow B$ is called an intuitionistic fuzzy homomorphism (or IF homomorphism) from A to $B$ if 


$$
\mu_{B}(f(x)) \geq \mu_{A}(x) \text { and } \nu_{B}(f(x)) \leq \nu_{A}(x), \forall x \in G \text {. }
$$

Given an IF homomorphism $\bar{f}: A \rightarrow B, f: G \rightarrow H$ is called the underlying homomorphism of $\bar{f}$. The set of all IF homomorphisms from $A$ to $B$ is denoted by $\operatorname{Hom}(A, B)$.

If $\bar{f}: A \rightarrow B$ is an IF homomorphism, we define that $\operatorname{Ker} \bar{f}=\left\{x \in G: \mu_{A}(f(x))=\right.$ $\left.1 ; \nu_{A}(f(x))=0\right\}$ and that $\operatorname{Im} \bar{f}=\{\bar{f}(x): x \in G\}$. If $f: G \rightarrow H$ is a homomorphism and Ker $f$ is the pre-image of $\{\theta\}$ under $f$ (where $\theta$ is zero element of $G$ ), we have $\operatorname{Ker} f \subseteq \operatorname{Ker} \bar{f}$. Especially, if $B=\chi_{H}$, then we have $\operatorname{Ker} \bar{f}=A$, for all $f \in \operatorname{Hom}(A, B)$.

Proposition 2. If $\bar{f}: A \rightarrow B$ is an intuitionistic fuzzy homomorphism, where $A$ and $B$ are IFSG of group $G$ and $H$, respectively, then we have the following:

(i) Ker $\bar{f}$ is a subgroup of $G$;

(ii) The restriction of $A$ to $\operatorname{Ker} \bar{f}$, i.e., $\left.A\right|_{\operatorname{Ker} \bar{f}}$ is an IFSG of $A$.

Proof. (i) Since $\bar{f}: A \rightarrow B$ is an intuitionistic fuzzy homomorphism. Let $\theta$ be zero element of $G$, then $\theta \in \operatorname{Ker} \bar{f}$. Further, if $x, y \in \operatorname{Ker} \bar{f}$, then we can easily show that $x+y \in \operatorname{Ker} \bar{f}$. This proves that $\operatorname{Ker} \bar{f}$ is a subgroup of $G$.

(ii) Let $C=\left.A\right|_{\operatorname{Ker} \bar{f}}$. Then $C=\left(\mu_{C}, \nu_{C}\right)$, where $\mu_{C}(x)=\mu_{A}(x)$ and $\nu_{C}(x)=\nu_{A}(x), \forall x \in$ Ker $\bar{f}$. Now, it is easy to show that $C$ is an IFSG of $G$ and $C \subseteq A$.

\section{Categories of intuitionistic fuzzy groups}

In this section we show that there exists a covariant functor from the category of ordinary groups to the category of intuitionistic fuzzy groups.

Proposition 3. Let $\operatorname{Hom}(A, B)$ be the set of all IF homomorphisms from the IFSG A of group $G$ into the IFSG $B$ of group $H$. Then $\operatorname{Hom}(A, B)$ is an Abelian additive group.

Proof. Since $\mu_{B}(\overline{0}(x))=\mu_{B}(0)=1 \geq \mu_{A}(x)$ and $\nu_{B}(\overline{0}(x))=\nu_{B}(0)=0 \leq \nu_{A}(x)$ implies that there exist zero IF homomorphism $\overline{0}: A \rightarrow B$. Let $\bar{f}, \bar{g} \in \operatorname{Hom}(A, B)$ and $\forall x \in G$, we have $\mu_{B}((f+g)(x))=\mu_{B}(f(x)+g(x)) \geq \mu_{B}(f(x)) \wedge \mu_{B}(g(x)) \geq \mu_{A}(x) \wedge \mu_{A}(x)=\mu_{A}(x)$.

Similarly, we can show that $\nu_{B}((f+g)(x)) \leq \nu_{A}(x)$. This shows that $\overline{f+g} \in \operatorname{Hom}(A, B)$. Now, we can define $\bar{f}+\bar{g}=\overline{f+g} \in \operatorname{Hom}(A, B)$. The addition obviously satisfies the commutative law and associative law. Also, define $-\bar{f}=\overline{-f}$ for every $\bar{f} \in \operatorname{Hom}(A, B)$.

We have confidence in the definition, because:

$\mu_{B}((-f)(x))=\mu_{B}(-(f(x)))=\mu_{B}(f(x)) \geq \mu_{A}(x)$ and $\nu_{B}((-f)(x))=\nu_{B}(-(f(x)))=$ $\nu_{B}(f(x)) \leq \nu_{A}(x), \forall x \in G$. This shows that $\overline{-f} \in \operatorname{Hom}(A, B), \forall f \in \operatorname{Hom}(A, B)$.

Precisely, $\bar{f}+\overline{0}=\overline{0}+\bar{f}$ and $\bar{f}+\overline{-f}=\overline{-f}+\bar{f}=\overline{0}$. This shows that $-\bar{f}$ works as the additive inverse of $\bar{f}$ and $\overline{0}$ is the zero element (or additive identity) in $\operatorname{Hom}(A, B)$. Hence $\operatorname{Hom}(A, B)$ is an additive Abelian group. 
Theorem 1. Let $A=\left(\mu_{A}, \nu_{A}\right)$ and $B=\left(\mu_{B}, \nu_{B}\right)$ are two IF groups of group $G$ and $H$, respectively. Then the function $\beta: \operatorname{Hom}(A, B) \rightarrow[0,1] \times[0,1]$ on group $\operatorname{Hom}(A, B)$ defined by

$$
\beta(\bar{f})=\left(\mu_{\beta(\bar{f})}, \nu_{\beta(\bar{f})}\right),
$$

where $\mu_{\beta(\bar{f})}=\wedge\left\{\mu_{B}(\bar{f}(x)): x \in G\right\}$ and $\nu_{\beta(\bar{f})}=\vee\left\{\nu_{B}(\bar{f}(x)): x \in G\right\}$ is an intuitionistic fuzzy subgroup of $\operatorname{Hom}(A, B)$.

Proof. As shown in Proposition 3, $\operatorname{Hom}(A, B)$ is an Abelian group. Next, we show that the function $\beta: \operatorname{Hom}(A, B) \rightarrow[0,1] \times[0,1]$ on group $\operatorname{Hom}(A, B)$ defined by

$$
\beta(\bar{f})=\left(\mu_{\beta(\bar{f})}, \nu_{\beta(\bar{f})}\right)
$$

where $\mu_{\beta(\bar{f})}=\wedge\left\{\mu_{B}(\bar{f}(x)): x \in G\right\}$ and $\nu_{\beta(\bar{f})}=\vee\left\{\nu_{B}(\bar{f}(x)): x \in G\right\}$ is an intuitionistic fuzzy subgroup of $\operatorname{Hom}(A, B)$. Let $\bar{f}, \bar{g} \in \operatorname{Hom}(A, B)$. Then we have

$$
\begin{aligned}
\mu_{\beta(\bar{f}+\bar{g})} & =\wedge\left\{\mu_{B}((\bar{f}+\bar{g})(x)): x \in G\right\} \\
& =\wedge\left\{\mu_{B}(\bar{f}(x)+\bar{g}(x)): x \in G\right\} \\
& \geq \wedge\left\{\left\{\mu_{B}(\bar{f}(x) \wedge \bar{g}(x))\right\}: x \in G\right\} \\
& =\left\{\wedge\left\{\mu_{B}(\bar{f}(x)): x \in G\right\}\right\} \wedge\left\{\wedge\left\{\mu_{B}(\bar{g}(x)): x \in G\right\}\right\} \\
& =\mu_{\beta(\bar{f})} \wedge \mu_{\beta(\bar{g})} .
\end{aligned}
$$

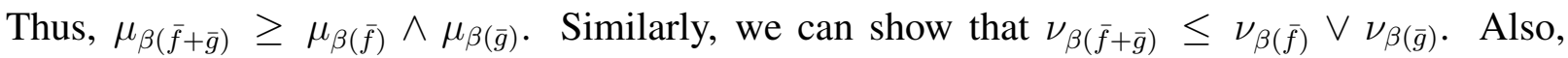
$\mu_{\beta(\overline{-f})}=\wedge\left\{\mu_{B}(\overline{-f}(x)): x \in G\right\}=\wedge\left\{\mu_{B}(-f(x)): x \in G\right\} \geq \wedge\left\{\mu_{B}(f(x)): x \in G\right\}=\mu_{\beta(\bar{f})}$. Similarly, we can show that $\nu_{\beta(\overline{-f})} \leq \nu_{\beta(\bar{f})}$. Hence $\beta$ is an intuitionistic fuzzy subgroup of group $\operatorname{Hom}(A, B)$.

Definition 12. Let $\boldsymbol{C}_{\boldsymbol{G}}=\left(O b\left(\boldsymbol{C}_{\boldsymbol{G}}\right), \operatorname{Hom}\left(\boldsymbol{C}_{\boldsymbol{G}}\right), i_{G}, o\right)$ be a category of groups and let $\operatorname{Hom}\left(\boldsymbol{C}_{\boldsymbol{G}}\right)=\bigcup\left\{\operatorname{Hom}_{\boldsymbol{C}_{\boldsymbol{G}}}(G, H): G, H \in O b\left(\boldsymbol{C}_{\boldsymbol{G}}\right)\right\}$. An IF-groups category $\boldsymbol{C}_{\boldsymbol{I F G}}$ over the base category $\boldsymbol{C}_{\boldsymbol{G}}$ is completely described by two mappings:

$$
\begin{gathered}
\alpha: O b\left(\boldsymbol{C}_{\boldsymbol{G}}\right) \rightarrow[0,1] \times[0,1] ; \\
\beta: \operatorname{Hom}\left(\boldsymbol{C}_{\boldsymbol{G}}\right) \rightarrow[0,1] \times[0,1] .
\end{gathered}
$$

IF-group category $\boldsymbol{C}_{I F G}$ consists of:

(Cl) a class $\mathrm{Ob}\left(\boldsymbol{C}_{\boldsymbol{G}}\right)$ of objects;

(C2) an IF subclass $\alpha$ of $\mathrm{Ob}\left(\boldsymbol{C}_{\boldsymbol{G}}\right), \alpha: \mathrm{Ob}\left(\boldsymbol{C}_{\boldsymbol{G}}\right) \rightarrow[0,1] \times[0,1]$;

(C3) a class $\operatorname{Hom}\left(\boldsymbol{C}_{\boldsymbol{G}}\right)=\bigcup\left\{\operatorname{Hom}_{\boldsymbol{C}_{\boldsymbol{G}}}(G, H): G, H \in \mathrm{Ob}\left(\boldsymbol{C}_{\boldsymbol{G}}\right)\right\}$ where $\operatorname{Hom}_{\boldsymbol{C}_{\boldsymbol{G}}}(G, H)$ are pairwise disjoint sets for each ordered object pair $(G, H)$;

(C4) an IF subclass $\beta$ of $\operatorname{Hom}\left(\boldsymbol{C}_{\boldsymbol{G}}\right), \beta: \operatorname{Hom}\left(\boldsymbol{C}_{\boldsymbol{G}}\right) \rightarrow[0,1] \times[0,1]$, such that if $f \in \operatorname{Hom}_{\boldsymbol{C}_{\boldsymbol{G}}}(G, H)$, then

$$
\beta(\bar{f})=\left(\mu_{\beta(\bar{f})}, \nu_{\beta(\bar{f})}\right)
$$

as defined in Theorem 1. 
(C5) a composition law associating to each pair of morphisms $f \in \operatorname{Hom}(G, H)$ and $g \in \operatorname{Hom}(H, P)$, a morphism $g \circ f \in \operatorname{Hom}(P, Q)$, such that the following axioms holds;

(G1) Associativity: $h \circ(g \circ f)=(h \circ g) \circ f$, for all $f \in \operatorname{Hom}(G, H), g \in \operatorname{Hom}(H, P)$ and $h \in \operatorname{Hom}(P, Q)$;

(G2) preservation of morphisms: $\beta(g \circ f)=\beta(g) \circ \beta(f)$;

(G3) existence of identity: for each $G \in \operatorname{ob}\left(\boldsymbol{C}_{\boldsymbol{G}}\right)$ there exists an identity $i_{G} \in \operatorname{Hom}_{\boldsymbol{C}_{\boldsymbol{G}}}(G, G)$ such that $\beta\left(i_{G}\right)=\alpha(G)$.

Remark 4. A category of IF groups $\boldsymbol{C}_{\boldsymbol{I F G}}=\left(O b\left(\boldsymbol{C}_{\boldsymbol{G}}\right), \alpha, H o m\left(\boldsymbol{C}_{\boldsymbol{G}}\right), \beta, \circ\right)$ can be constructed as

$$
\boldsymbol{C}_{\boldsymbol{I F G}}=\left(O b\left(\boldsymbol{C}_{\boldsymbol{I F G}}\right), \operatorname{Hom}\left(\boldsymbol{C}_{\boldsymbol{I F G}}\right), \circ\right)
$$

consisting of two classes:

(i) a class of objects $=O b\left(\boldsymbol{C}_{\boldsymbol{I F G}}\right)=\left\{\alpha(G): G \in O b\left(\boldsymbol{C}_{\boldsymbol{G}}\right)\right\}$ forms an IF subclass of $\mathrm{Ob}\left(\boldsymbol{C}_{\boldsymbol{G}}\right)$;

(ii) a class of morphisms $\operatorname{Hom}\left(\boldsymbol{C}_{\boldsymbol{I F G}}\right)=\left\{\beta(f): f \in \operatorname{Hom}\left(\boldsymbol{C}_{\boldsymbol{G}}\right)\right\}$ forms an IF subclass of $\operatorname{Hom}\left(\boldsymbol{C}_{\boldsymbol{G}}\right)$;

Proposition 4. $\boldsymbol{C}_{G}$ is a subcategory of $\boldsymbol{C}_{I F G}$.

Proof. It follows from (2.3), (2.7) and (3.4).

Proposition 5. There exists a covariant functor from $\boldsymbol{C}_{\boldsymbol{G}}$ to $\boldsymbol{C}_{\boldsymbol{I F G}}$.

Proof. Define $\beta=\left(\mu_{\beta}, \nu_{\beta}\right): \mathbf{C}_{\mathbf{G}} \rightarrow \mathbf{C}_{\mathbf{I F G}}$ by $\beta(G)=\left(\mu_{\beta}(G), \nu_{\beta}(G)\right)$, where $\mu_{\beta}(x)+\nu_{\beta}(x) \leq$ $1, \forall x \in G$.

Let $f \in \operatorname{Hom}_{\mathbf{C}_{\mathbf{G}}}(G, H)$. Then $\beta(f) \in \operatorname{Hom}\left(\mathbf{C}_{\mathbf{I F G}}\right)$, where $\beta(f): \beta(G) \rightarrow \beta(H)$ defined by

$$
\beta(f)\left(\mu_{\beta}, \nu_{\beta}\right)=\left(\mu_{\beta} \circ f^{-1}, \nu_{\beta} \circ f^{-1}\right) \text {, where }
$$

(i) $\mu_{\beta}(x+y) \geq \mu_{\beta}(x) \wedge \mu_{\beta}(y)$

(ii) $\nu_{\beta}(x+y) \leq \nu_{\beta}(x) \vee \nu_{\beta}(y)$

(iii) $\mu_{\beta}(-x)=\mu_{\beta}(x)$

(iv) $\nu_{\beta}(-x)=\nu_{\beta}(x), \forall x, y \in G$.

We want to prove that $\beta$ preserves objects, composition, domains and codomain and identity.

Let $\left(\mu_{\beta}, \nu_{\beta}\right),\left(\mu_{\beta_{1}}, \nu_{\beta_{1}}\right) \in O b\left(\mathbf{C}_{\mathbf{I F G}}\right)$ such that $\beta(f)\left(\mu_{\beta}, \nu_{\beta}\right)=\beta(f)\left(\mu_{\beta_{1}}, \nu_{\beta_{1}}\right)$

$\Rightarrow\left(\mu_{\beta} \circ f^{-1}, \nu_{\beta} \circ f^{-1}\right)=\left(\mu_{\beta_{1}} \circ f^{-1}, \nu_{\beta_{1}} \circ f^{-1}\right)$

$\Rightarrow \mu_{\beta} \circ f^{-1}=\mu_{\beta_{1}} \circ f^{-1}$ and $\nu_{\beta} \circ f^{-1}=\nu_{\beta_{1}} \circ f^{-1}$

$\Rightarrow \mu_{\beta}=\mu_{\beta_{1}}$ and $\nu_{\beta}=\nu_{\beta_{1}} \Rightarrow\left(\mu_{\beta}, \nu_{\beta}\right)=\left(\mu_{\beta_{1}}, \nu_{\beta_{1}}\right)$

$\Rightarrow \beta$ is well defined.

Let $f \in \operatorname{Hom}_{\mathbf{C}_{\mathbf{G}}}(G, H), g \in \operatorname{Hom}_{\mathbf{C}_{\mathbf{G}}}(H, P)$ then $g \circ f \in \operatorname{Hom}_{\mathbf{C}_{\mathbf{G}}}(G, P)$. 
Also, then $\beta(f) \in \operatorname{Hom}_{\mathbf{C}_{\text {IFG }}}(\beta(G), \beta(H)), \beta(g) \in \operatorname{Hom}_{\mathbf{C}_{\mathbf{I F G}}}(\beta(H), \beta(P))$ and $\beta(g \circ f) \in$ $\operatorname{Hom}_{\mathbf{C}_{\mathbf{I F G}}}(\beta(G), \beta(P))$. Let $\left(\mu_{\beta}, \nu_{\beta}\right) \in \beta(G)$, then we have

$$
\begin{aligned}
\beta(g \circ f)\left(\mu_{\beta}, \nu_{\beta}\right) & =\left(\mu_{\beta} \circ(g \circ f)^{-1}, \nu_{\beta} \circ(g \circ f)^{-1}\right) \\
& =\left(\mu_{\beta} \circ\left(f^{-1} \circ g^{-1}\right), \nu_{\beta} \circ\left(f^{-1} \circ g^{-1}\right)\right) \\
& =\left(\left(\mu_{\beta} \circ f^{-1}\right) \circ g^{-1},\left(\nu_{\beta} \circ f^{-1}\right) \circ g^{-1}\right) \\
& =\beta(g)\left(\mu_{\beta} \circ f^{-1}, \nu_{\beta} \circ f^{-1}\right) \\
& =\beta(g) \beta(f)\left(\mu_{\beta}, \nu_{\beta}\right) .
\end{aligned}
$$

Therefore, $\beta(g \circ f)=\beta(g) \beta(f)$.

Also, $\beta\left(i_{G}\right)\left(\mu_{\beta}, \nu_{\beta}\right)=\left(\mu_{\beta} \circ i_{G}^{-1}, \nu_{\beta} \circ i_{G}^{-1}\right)=\left(\mu_{\beta}, \nu_{\beta}\right)$ implies that $\beta\left(i_{G}\right)$ is the identity element in $\operatorname{Hom}\left(\mathbf{C}_{\mathbf{I F G}}\right)$. Hence $\beta: \mathbf{C}_{\mathbf{G}} \rightarrow \mathbf{C}_{\mathbf{I F G}}$ is a covariant functor.

\section{Optimal intuitionistic fuzzification}

In this section we show that the category $\mathbf{C}_{\mathbf{I F G}}$ form a top category over the category $\mathbf{C}_{\mathbf{G}}$. To prove this we first construct a category $\mathbf{C}_{\mathbf{L a t}(\mathbf{G})}$ of complete lattices corresponding to every object in $\mathbf{C}_{\mathbf{G}}$ and then show that corresponding to each morphism in $\mathbf{C}_{\mathbf{G}}$ there exist a contravariant functor from $\mathbf{C}_{\mathbf{I F G}}$ to the category $\mathbf{C}_{\mathbf{L a t}}$ (= union of all $\mathbf{C}_{\mathbf{L a t}(\mathbf{G})}$, corresponding to each object in $\mathbf{C}_{\mathbf{G}}$ ) that preserves infima. Finally, we define the notion of kernel and cokernel for the category $\mathbf{C}_{\mathbf{I F G}}$ and show that $\mathbf{C}_{\mathbf{I F G}}$ is not an Abelian category.

Let $A=\left(\mu_{A}, \nu_{A}\right)$ be an IFSG of $G$, let $H$ be a group and let $f: G \rightarrow H$ be a group homomorphism. With the help of $A$ and $f$, we can provide an IF group structure on $H$ by

$$
\mu_{f(A)}(y)=\sup \left\{\mu_{A}(x): f(x)=y\right\} \text { and } \nu_{f(A)}(y)=\inf \{\nu(x): f(x)=y\} .
$$

It is clear that $f(A)=\left(\mu_{f(A)}, \nu_{f(A)}\right)$ is an IFSG of $H$ and $\bar{f}: A \rightarrow f(A)$ is a homomorphism of intuitionistic fuzzy groups.

If $G$ is a group and $B=\left(\mu_{B}, \nu_{B}\right)$ is an IFSG of $H$ and $f: G \rightarrow H$ is a group homomorphism, then we can define IF group structure on $G$ by

$$
\mu_{f^{-1}(B)}(x)=\mu_{B}(f(x)) \text { and } \nu_{f^{-1}(B)}(x)=\nu_{B}(f(x)) .
$$

Hence, $f^{-1}(B)=\left(\mu_{f^{-1}(B)}, \nu_{f^{-1}(B)}\right)$ is an IFSG of $G$ and $\bar{f}: f^{-1}(B) \rightarrow B$ is a homomorphism of intuitionistic fuzzy groups.

Lemma 1. Let $G$ and $H$ be groups and $f: G \rightarrow H$ be a group homomorphism.

(i) If $A=\left(\mu_{A}, \nu_{A}\right)$ is an IFSG of $G$, then there exists an IFSG $f(A)=\left(\mu_{f(A)}, \nu_{f(A)}\right)$ of $H$ such that for any IFSG $\left(\mu_{B}, \nu_{B}\right)$ of $H, \bar{f}: A \rightarrow B$ is an IF homomorphism if and only if $f(A) \subseteq B$.

(ii) If $B=\left(\mu_{B}, \nu_{B}\right)$ is an IFSG of $H$, then there exists an IFSG $f^{-1}(B)=\left(\mu_{f^{-1}(B)}, \nu_{f^{-1}(B)}\right)$ of $G$ such that for any IFSG $A$ of $G, \bar{f}: A \rightarrow B$ is an IF homomorphism if and only if $A \subseteq f^{-1}(B)$. 
Proof. (i) Now, $\bar{f}: A \rightarrow B$ is an IF homomorphism if and only if $\mu_{B}(f(x)) \geq \mu_{A}(x)$ and $\nu_{B}(f(x)) \leq \nu_{A}(x), \forall x \in G$. Let $y \in H$ be any element, then $\mu_{f(A)}(y)=\vee\left\{\mu_{A}(x): f(x)=y\right\}$ $\leq \mu_{A}(x) \leq \mu_{B}(f(x))$.

Similarly, we can show that $\nu_{f(A)}(y) \geq \nu_{B}(f(x))$ i.e., $f(A) \subseteq B$.

(ii) Now, $\bar{f}: A \rightarrow B$ is an IF homomorphism if and only if $\mu_{B}(f(x)) \geq \mu_{A}(x)$ and $\nu_{B}(f(x)) \leq \nu_{A}(x), \forall x \in G$. Now, $\mu_{f^{-1}(B)}(x)=\mu_{B}(f(x)) \geq \mu_{A}(x)$ and $\nu_{f^{-1}(B)}(x)=$ $\nu_{B}(f(x)) \leq \nu_{A}(x)$ implies that $A \subseteq f^{-1}(B)$.

Notice that if $f: G \rightarrow H$ is a homomorphism, then for every IFSG $A[B]$ of $G[H]$ one has $f(A)\left[f^{-1}(B)\right]$ IFSGs, we say that $f$ is trivially intuitionistic fuzzified relative to $A[B]$. In particular, if $f: G \rightarrow H$ is a homomorphism, then for each IFSG $A[B]$ of $G[H]$, we have IF homomorphism $\bar{f}: A \rightarrow \chi_{H}\left[\bar{f}: \chi_{G} \rightarrow B\right]$.

Lemma 2. The set $s(G)=\left\{\left(\mu_{A}, \nu_{A}\right): G \rightarrow[0,1] \times[0,1]:\left(\mu_{A}, \nu_{A}\right)\right.$ is IF group of group $\left.G\right\}$ form a complete lattice associated with the order relation $\left(\mu_{1}, \nu_{1}\right) \leq\left(\mu_{2}, \nu_{2}\right)$ if $\mu_{1}(x) \leq \mu_{2}(x)$ and $\nu_{1}(x) \geq \nu_{2}(x), \forall x \in G$.

Proof. Let $\left\{\left(\mu_{i}, \nu_{i}\right): i \in J\right\}$ be a collection of elements of $s(G)$. Then infimum and supremum on $s(G)$ are defined as follows:

$$
\wedge_{i \in J}\left(\mu_{i}, \nu_{i}\right)(x)=\left(\inf _{i \in J}\left\{\mu_{i}(x)\right\}, \sup _{i \in J}\left\{\nu_{i}(x)\right\}\right)
$$

and

$\vee_{i \in J}\left(\mu_{i}, \nu_{i}\right)(x)=\left(\inf _{i \in J}\left\{\mu(x):\left(\mu_{i}, \nu_{i}\right) \in s(G)\right.\right.$ and $\left.\mu_{i} \leq \mu\right\}, \sup _{i \in J}\left\{\nu(x):\left(\mu_{i}, \nu_{i}\right) \in s(G)\right.$ and $\left.\left.\nu_{i} \geq \nu\right\}\right)$.

Then $s(G)$ form a complete lattice.

\section{Remark 5.}

(i) The least element of $s(G)$ is $\overline{0}$ and the greatest elements of $s(G)$ is $\overline{1}$.

(ii) $s(G)$ under the order relation defined above form a category where $O b(s(G))=$ all IF-group on $G$ and $\operatorname{Hom}(s(G))=$ order relation defined above.

(iii) Supremum can also be defined as $\vee_{i \in J}\left(\mu_{i}, \nu_{i}\right)(x)=\left(\sup _{i \in J}\left\{\mu_{i}(x)\right\}, \inf _{i \in J}\left\{\nu_{i}(x)\right\}\right)$ which only holds for IF sets but does not hold for IF groups including when $J$ is finite.

For example, let $G=(Z,+)$ and IFSGs $(\mu, \nu)$ and $\left(\mu^{\prime}, \nu^{\prime}\right)$ on $G$ defined as:

$$
(\mu, \nu)(x)=\left\{\begin{array}{ll}
(1,0), & \text { if } x \text { is even } \\
(0,1), & \text { if } x \text { is odd }
\end{array} ; \quad\left(\mu^{\prime}, \nu^{\prime}\right)(x)=\left\{\begin{array}{ll}
(1,0), & \text { if } 3 \mid x \\
(0,1), & \text { otherwise }
\end{array} .\right.\right.
$$

Take $(\mu, \nu) \vee\left(\mu^{\prime}, \nu^{\prime}\right)=\left(\mu^{\prime \prime}, \nu^{\prime \prime}\right)$, where $\mu^{\prime \prime}(x)=\max \left\{\mu(x), \mu^{\prime}(x)\right\}, \nu^{\prime \prime}(x)=\min \left\{\nu(x), \nu^{\prime}(x)\right\}$. Here, we can check that $\left(\mu^{\prime \prime}, \nu^{\prime \prime}\right)$ is not IFSG of $G$, for $0=\mu^{\prime \prime}(1)=\mu^{\prime \prime}(3-2)<\mu^{\prime \prime}(3) \wedge \mu^{\prime \prime}(2)=1$ and $1=\nu^{\prime \prime}(1)=\nu^{\prime \prime}(3-2)>\nu^{\prime \prime}(3) \vee \nu^{\prime \prime}(2)=0$.

Lemma 3. The set $t(G)=\left\{\left(\mu_{A}, \nu_{A}\right): G \rightarrow[0,1] \times[0,1]:\left(\mu_{A}, \nu_{A}\right)\right.$ is IF group on group $\left.G\right\}$ form a complete lattice associated with the order relation $\left(\mu_{1}, \nu_{1}\right) \leq\left(\mu_{2}, \nu_{2}\right)$ if $\mu_{1}(x) \geq \mu_{2}(x)$ and $\nu_{1}(x) \leq \nu_{2}(x) \forall x \in G$. 
Proof. Let $\left\{\left(\mu_{i}, \nu_{i}\right): i \in J\right\}$ be a collection of elements of $t(G)$. Then infimum and supremum on $t(G)$ are defined as follows:

$$
\wedge_{i \in J}\left(\mu_{i}, \nu_{i}\right)(x)=\left(\sup _{i \in J}\left\{\mu_{i}(x)\right\}, \inf _{i \in J}\left\{\nu_{i}(x)\right\}\right)
$$

and

$\vee_{i \in J}\left(\mu_{i}, \nu_{i}\right)(x)=\left(\inf _{i \in J}\left\{\mu(x):\left(\mu_{i}, \nu_{i}\right) \in t(G)\right.\right.$ and $\left.\mu_{i} \leq \mu\right\}, \sup _{i \in J}\left\{\nu(x):\left(\mu_{i}, \nu_{i}\right) \in t(G)\right.$ and $\left.\left.\nu_{i} \geq \nu\right\}\right)$.

Then $t(G)$ form a complete lattice.

Remark 6. $t(G)$ under the order relation defined above form a category where $O b(t(G))=$ all IF-groups on $G$ and $\operatorname{Hom}(t(G))=$ order relation as defined above.

Theorem 2. $\boldsymbol{C}_{I F G}$ is a top category over $\boldsymbol{C}_{\boldsymbol{G}}$.

Proof. It suffices to show that assigning to each $G \in O b\left(\mathbf{C}_{\mathbf{G}}\right)$ the corresponding complete lattice $s(G)$ defined in Lemma 2 and to every $f \in \operatorname{Hom}_{\mathbf{C}_{\mathbf{G}}}(G, H)$ the function $s(f): s(H) \rightarrow$ $s(G)$ defined as $s(f)\left(\mu_{B}, \nu_{B}\right)=\left(\mu_{f^{-1}(B)}, \nu_{f^{-1}(B)}\right), \forall\left(\mu_{B}, \nu_{B}\right) \in s(H)$ determine a contravarient functor $s: \mathbf{C}_{\mathbf{I F G}} \rightarrow \mathbf{C}_{\mathbf{L a t}}$. In other words, we need to show that

(i) for all $f \in \operatorname{Hom}_{\mathbf{C}_{\mathbf{G}}}(G, H), s(f)$ preserves infima,

(ii) for each $f, g \in \operatorname{Hom}_{\mathbf{C}_{\mathbf{G}}}(G, H)$ we have $s(g \circ f)=s(f) \circ s(g)$ and

(iii) $s\left(i_{G}\right): s(G) \rightarrow s(G)$ is the identity function for each identity homomorphism $i_{G}: G \rightarrow G$.

Consider $\left\{\left(\mu_{B_{i}}, \nu_{B_{i}}\right): i \in J\right\} \subset s(H)$ to be a non-empty subfamily of $s(H)$ and let $x \in G$. Then

$$
\begin{aligned}
s(f)\left[\wedge\left(\mu_{B_{i}}, \nu_{B_{i}}\right)\right](x) & =\left(\inf \left\{\mu_{f^{-1}\left(B_{i}\right)}\right\}, \sup \left\{\nu_{f^{-1}\left(B_{i}\right)}\right\}\right)(x) \\
& =\left(\inf \left\{\mu_{f^{-1}\left(B_{i}\right)}(x)\right\}, \sup \left\{\nu_{f^{-1}\left(B_{i}\right)}(x)\right\}\right) \\
& =\left(\inf \left\{\mu_{B_{i}}(f(x))\right\}, \sup \left\{\nu_{B_{i}}(f(x))\right\}\right) \\
& =\left(\inf \left\{\mu_{B_{i}}\right\}, \sup \left\{\nu_{B_{i}}\right\}\right)(f(x)) \\
& =\wedge\left(\mu_{B_{i}}, \nu_{B_{i}}\right)(f(x)) \\
& =\wedge\left(\mu_{B_{i}}(f(x)), \nu_{B_{i}}(f(x))\right) \\
& =\wedge\left(\mu_{f^{-1}\left(B_{i}\right)}(x), \nu_{f^{-1}\left(B_{i}\right)}(x)\right) \\
& =\wedge\left(\mu_{f^{-1}\left(B_{i}\right)}, \nu_{f^{-1}\left(B_{i}\right)}\right)(x) \\
& =\wedge\left[s(f)\left(\mu_{B_{i}}, \nu_{B_{i}}\right)\right](x) .
\end{aligned}
$$

Thus, $s(f)$ preserves infima. 
Let $f: G \rightarrow H, g: H \rightarrow T$ be homomorphisms and let $\left(\mu_{C}, \nu_{C}\right) \in s(T)$ and $x \in G$, then

$$
\begin{aligned}
s(g \circ f)\left(\mu_{C}, \nu_{C}\right)(x) & =\left(\mu_{(g \circ f)^{-1}(C)}, \nu_{(g \circ f)^{-1}(C)}\right)(x) \\
& =\left(\mu_{\left(f^{-1} \circ g^{-1}\right)(C)}(x), \nu_{\left(f^{-1} \circ g^{-1}\right)(C)}(x)\right) \\
& =\left(\mu_{\left(f^{-1}\left(g^{-1}(C)\right)\right)}(x), \nu_{\left(f^{-1}\left(g^{-1}(C)\right)\right)}(x)\right) \\
& =s(f)\left(\mu_{g^{-1}(C)}(x), \nu_{g^{-1}(C)}(x)\right) \\
& =s(f)\left(s(g)\left(\mu_{C}(x), \nu_{C}(x)\right)\right) \\
& =s(f) s(g)\left(\mu_{C}, \nu_{C}\right)(x) .
\end{aligned}
$$

Thus, $s(g \circ f)=s(f) s(g)$.

Further, let $i_{G}: G \rightarrow G$ be the identity homomorphism defined by $i_{G}(x)=x, \forall x \in G$. Then $s\left(i_{G}\right)$ is the identity element in $\operatorname{Hom}\left(\mathbf{C}_{\mathbf{I F G}}\right)$, for if $\left(\mu_{A}, \nu_{A}\right) \in s(G)$ be any element, then $s\left(i_{G}\right)\left(\mu_{A}, \nu_{A}\right)(x)=\left(\mu_{i_{G}^{-1}(A)}(x), \nu_{i_{G}^{-1}(A)}(x)\right)=\left(\mu_{i_{G}(A)}(x), \nu_{i_{G}(A)}(x)\right)=\left(\mu_{A}(x), \nu_{A}(x)\right)=$ $\left(\mu_{A}, \nu_{A}\right)(x)$.

This completes the proof.

Remark 7. There exists a covariant functor $t: \boldsymbol{C}_{\boldsymbol{I F G}} \rightarrow \boldsymbol{C}_{\boldsymbol{L a t}}$ such that $t(f): t(G) \rightarrow t(H)$ preserves suprema and is defined by $t(f)\left(\mu_{A}, \nu_{A}\right)=\left(\mu_{f(A)}, \nu_{f(A)}\right), \forall\left(\mu_{A}, \nu_{A}\right) \in t(G)$ such that $t(g \circ f)=t(g) \circ t(f), \forall f: G \rightarrow H, g: H \rightarrow T$.

Proof. It is easy to check that $t(f)$ preserves suprema and $t\left(i_{G}\right)$ is the identity element in $\operatorname{Hom}\left(\mathbf{C}_{\mathbf{I F G}}\right)$. Furthermore, we have

$$
\begin{aligned}
t(g \circ f)\left(\mu_{A}, \nu_{A}\right)(z) & =\left(\mu_{(g \circ f)(A)}(z), \nu_{(g \circ f)(A)}(z)\right) \\
& =\left(\mu_{g(f(A))}(z), \nu_{g(f(A))}(z)\right) \\
& =t(g)\left(\mu_{f(A)}(z), \mu_{f(A)}(z)\right) \\
& =t(g)\left(t(f)\left(\mu_{A}(z), \nu_{A}(z)\right)\right) \\
& =t(g) t(f)\left(\mu_{A}(z), \nu_{A}(z)\right) \\
& =t(g) t(f)\left(\mu_{A}, \nu_{A}\right)(z) .
\end{aligned}
$$

Thus $t(g \circ f)=t(g) t(f)$. This completes the proof.

Lemma 4. (i) Let $\left\{G_{i}: i \in J\right\}, H$ be groups and $\mathfrak{A}=\left\{f_{i}: G_{i} \rightarrow H: i \in J\right\}$ be a collection of homomorphisms. If $\left\{A_{i}: i \in J\right\}$ is a collection of IFSGs of $G_{i}$, then there exists a smallest IFSG $B=\left(\mu_{B}, \nu_{B}\right)$ of $H$ such that $\bar{f}_{i}: A_{i} \rightarrow B$ is an IF homomorphism, $\forall i \in J$, where $\left(\mu_{B}, \nu_{B}\right)=$ $(\mu, \nu)^{\mathfrak{A}}=\left(\mu^{\mathfrak{A}}, \nu^{\mathfrak{A}}\right)$, here $\mu_{B}=\mu^{\mathfrak{A}}=\vee\left\{\mu_{f_{i}\left(A_{i}\right)}: i \in J\right\}$ and $\nu_{B}=\nu^{\mathfrak{A}}=\wedge\left\{\nu_{f_{i}\left(A_{i}\right)}: i \in J\right\}$.

(ii) Let $G$ and $\left\{H_{i}: i \in J\right\}$ be groups and $\mathfrak{B}=\left\{g_{i}: G \rightarrow H_{i}: i \in J\right\}$ be a collection of homomorphisms. If $\left\{B_{i}: i \in J\right\}$ are IFSGs of $H_{i}$, then there exists a largest IFSG $A=\left(\mu_{A}, \nu_{A}\right)$ of $G$ such that $\bar{g}_{i}: A \rightarrow B_{i}$ is an IF homomorphism, $\forall i \in J$, where $\left(\mu_{A}, \nu_{A}\right)=(\mu, \nu)_{\mathfrak{B}}=$ $\left(\mu_{\mathfrak{B}}, \nu_{\mathfrak{B}}\right)$, here $\mu_{A}=\mu_{\mathfrak{B}}=\wedge\left\{\mu_{g_{i}^{-1}\left(B_{i}\right)}: i \in J\right\}$ and $\nu_{A}=\nu_{\mathfrak{B}}=\vee\left\{\nu_{g_{i}^{-1}\left(B_{i}\right)}: i \in J\right\}$.

Proof. (i) Using Lemma 1(i), for each $i \in J, A_{i}$ is IFSG of $G_{i}$ there exist IFSG $f_{i}\left(A_{i}\right)$ on $H$ such that for any IFSG $B=\left(\mu_{B}, \nu_{B}\right)$ of $H, \bar{f}_{i}: A_{i} \rightarrow B$ is an IF homomorphism if and only if $f_{i}\left(A_{i}\right) \subseteq B$, i.e., $\mu_{B} \geq \mu_{f_{i}\left(A_{i}\right)}$ and $\nu_{B} \leq \nu_{f_{i}\left(A_{i}\right)}$. 
Let $\mu^{\mathfrak{A}}=\vee\left\{\mu_{f_{i}\left(A_{i}\right)}: i \in J\right\}$ and $\nu^{\mathfrak{A}}=\wedge\left\{\nu_{f_{i}\left(A_{i}\right)}: i \in J\right\}$. Then the result follows.

(ii) Using Lemma 1(ii), for each $i \in J, B_{i}$ is IFSGs of $H$, then there exists an IFSG $g_{i}^{-1}\left(B_{i}\right)$ of $G$ such that for any IFSG $A=\left(\mu_{A}, \nu_{A}\right)$ of $G, \bar{g}_{i}: A \rightarrow B_{i}$ is an IF homomorphism if and only if $A \subseteq g_{i}^{-1}\left(B_{i}\right)$, i.e., $\mu_{A} \leq \mu_{g_{i}^{-1}\left(B_{i}\right)}$ and $\nu_{A} \geq \nu_{g_{i}^{-1}\left(B_{i}\right)}$.

Let $\mu_{\mathfrak{B}}=\wedge\left\{\left(\mu_{g_{i}^{-1}\left(B_{i}\right)}: i \in J\right\}\right.$ and $\nu_{\mathfrak{B}}=\vee\left\{\left(\nu_{g_{i}^{-1}\left(B_{i}\right)}: i \in J\right\}\right.$. Then the result follows.

Lemma 5. (i) Let $\left\{A_{i}: i \in J\right\}$ be a collection of IFSGs of group $G_{i}, i \in J$ and $\mathfrak{A}=\left\{f_{i}: G_{i} \rightarrow\right.$ $H: i \in J\}$ be a collection of homomorphisms and $g: H \rightarrow T$ a homomorphism then

$$
(\mu, \nu)^{\mathfrak{A}_{1}}=t(g)(\mu, \nu)^{\mathfrak{A}} \text {, where } \mathfrak{A}_{1}=\left\{g \circ f_{i}: G_{i} \rightarrow T: i \in J\right\} .
$$

(ii) Let $\left\{B_{i}: i \in J\right\}$ be a collection of IFSGs of $H_{i}, \forall i \in J$ and $\mathfrak{B}=\left\{g_{i}: G \rightarrow H_{i}: i \in J\right\}$ be a collection of homomorphisms and $h: L \rightarrow G$ be a homomorphism then

$$
(\mu, \nu)_{\mathfrak{B}_{1}}=s(h)(\mu, \nu)_{\mathfrak{B}} \text {, where } \mathfrak{B}_{1}=\left\{g_{i} \circ h: L \rightarrow H_{i}: i \in J\right\}
$$

Proof. (i) Let $\mathfrak{A}_{1}=\left\{g_{i}=g \circ f_{i}: G_{i} \rightarrow T: i \in J\right\}$ be the collection of homomorphisms. Then by Lemma 4(i), there exist IFSG $C=\left(\mu_{C}, \nu_{C}\right)$ of $T$ such that $g_{i}: A_{i} \rightarrow C$ is IF homomorphism, $\forall i \in J$, where $\left(\mu_{C}, \nu_{C}\right)=(\mu, \nu)^{\mathfrak{A}_{1}}=\left(\mu^{\mathfrak{A}_{1}}, \nu^{\mathfrak{A}_{1}}\right)$, here $\mu^{\mathfrak{A}_{1}}=\vee\left\{\mu_{g_{i}\left(A_{i}\right)}: i \in J\right\}$ and $\nu^{\mathfrak{A}_{1}}=\wedge\left\{\nu_{g_{i}\left(A_{i}\right)}: i \in J\right\}$. Now, we have

$$
\begin{aligned}
(\mu, \nu)^{\mathfrak{A}_{\perp}} & =\vee\left\{\left(\mu_{g_{i}\left(A_{i}\right)}, \nu_{g_{i}\left(A_{i}\right)}\right): i \in J\right\} \\
& =\vee\left\{\left(\mu_{\left(g \circ f_{i}\right)\left(A_{i}\right)}, \nu_{\left(g \circ f_{i}\right)\left(A_{i}\right)}\right): i \in J\right\} \\
& =\vee\left\{\left(\mu_{\left(g\left(f_{i}\left(A_{i}\right)\right)\right)}, \nu_{\left(g\left(f_{i}\left(A_{i}\right)\right)\right)}\right): i \in J\right\} \\
& =\vee\left\{t(g)\left(\mu_{f_{i}\left(A_{i}\right)}, \nu_{f_{i}\left(A_{i}\right)}\right): i \in J\right\} \\
& =t(g) \vee\left\{\left(\mu_{f_{i}\left(A_{i}\right)}, \nu_{f_{i}\left(A_{i}\right)}\right): i \in J\right\} \\
& =t(g)(\mu, \nu)^{\mathfrak{A}} .
\end{aligned}
$$

(ii) Let $\mathfrak{B}_{1}=\left\{h_{i}=g_{i} \circ h: L \rightarrow H_{i}: i \in J\right\}$ be the collection of homomorphisms. Then by Lemma 4(ii), there exists IFSG $A=\left(\mu_{A}, \nu_{A}\right)$ of $L$ such that $h_{i}: A \rightarrow C_{i}$ is IF homomorphism, $\forall i \in J$, where $\left(\mu_{A}, \nu_{A}\right)=(\mu, \nu)_{\mathfrak{B}_{1}}=\left(\mu_{\mathfrak{B}_{1}}, \nu_{\mathfrak{B}_{1}}\right)$, here $\mu_{\mathfrak{B}_{1}}=\wedge\left\{\mu_{h_{i}^{-1}\left(C_{i}\right)}: i \in J\right\}$ and $\nu_{\mathfrak{B}_{1}}=\vee\left\{\nu_{h_{i}^{-1}\left(C_{i}\right)}: i \in J\right\}$. Now, we have

$$
\begin{aligned}
(\mu, \nu)_{\mathfrak{B}_{1}} & =\wedge\left\{\left(\mu_{h_{i}^{-1}\left(C_{i}\right)}, \nu_{h_{i}^{-1}\left(C_{i}\right)}\right): i \in J\right\} \\
& =\wedge\left\{\left(\mu_{\left(g_{i} \circ h\right)^{-1}\left(C_{i}\right)}, \nu_{\left(g_{i} \circ h\right)^{-1}\left(C_{i}\right)}\right): i \in J\right\} \\
& =\wedge\left\{\left(\mu_{\left(h^{-1} \circ g_{i}^{-1}\right)\left(C_{i}\right)}, \nu_{\left(h^{-1} \circ g_{i}^{-1}\right)\left(C_{i}\right)}\right): i \in J\right\} \\
& =\wedge\left\{\left(\mu_{h^{-1}\left(g_{i}^{-1}\left(C_{i}\right)\right)}, \nu_{h^{-1}\left(g_{i}^{-1}\left(C_{i}\right)\right)}\right): i \in J\right\} \\
& =\wedge\left\{s(h)\left(\mu_{g_{i}^{-1}\left(C_{i}\right)}, \nu_{g_{i}^{-1}\left(C_{i}\right)}\right): i \in J\right\} \\
& =s(h) \wedge\left\{\left(\mu_{g_{i}^{-1}\left(C_{i}\right)}, \nu_{g_{i}^{-1}\left(C_{i}\right)}\right): i \in J\right\} \\
& =s(h)(\mu, \nu)_{\mathfrak{B}} .
\end{aligned}
$$

Thus, $(\mu, \nu)_{\mathfrak{B}_{1}}=s(h)(\mu, \nu)_{\mathfrak{B}}$. 
Remark 8. From Lemma 4 and Lemma 5, we are able to optimally intuitionistically fuzzified $f_{i}\left[g_{i}\right]$ in respect to family of IFSGs $\left\{A_{i}: i \in J\right\}\left[\left\{B_{i}: i \in J\right\}\right]$.

Theorem 3. The category of IF groups $\boldsymbol{C}_{I F G}$ has kernels and cokernels and is not an Abelian category.

Proof. Let $A=\left(\mu_{A}, \nu_{A}\right)$ and $B=\left(\mu_{B}, \nu_{B}\right)$ be IFSG of groups $G$ and $H$, respectively. Let $\bar{f}: A \rightarrow B$ be an IF homomorphism corresponding the homomorphism $f: G \rightarrow H$.

For Ker $f$, there exists an inclusion map $g: \operatorname{Ker} f \rightarrow G$ so that the following diagram commutes.
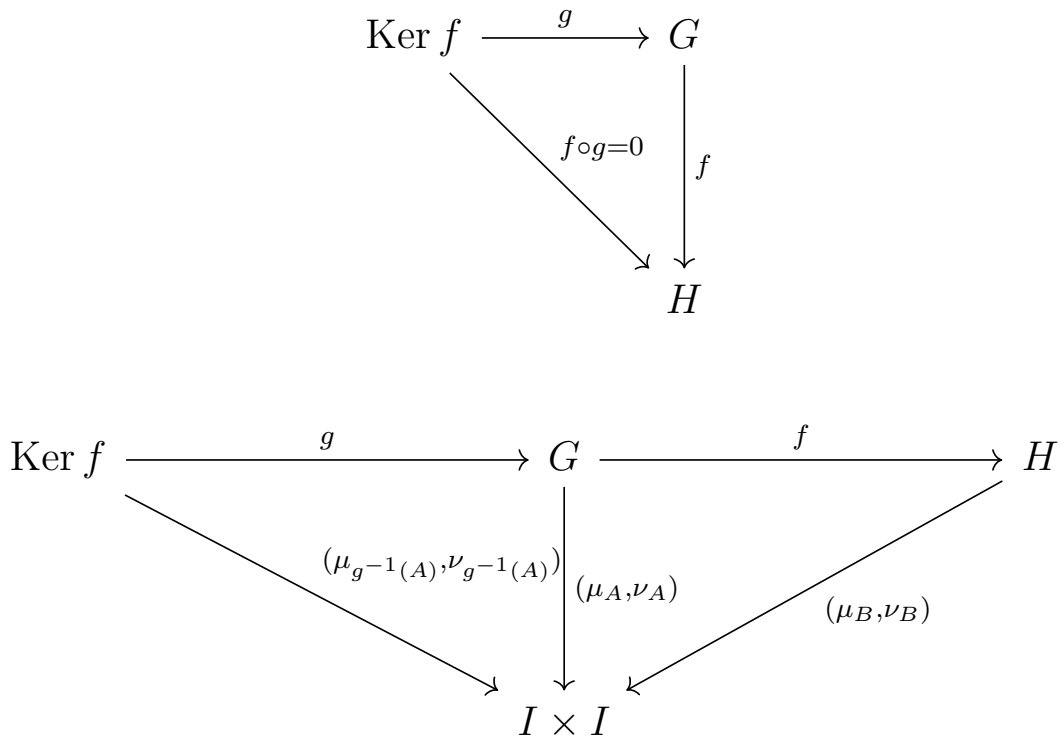

For Ker $\bar{f}$, there exists an inclusion map $\bar{g}: g^{-1}(A) \rightarrow A$ such that the following diagram commutes.

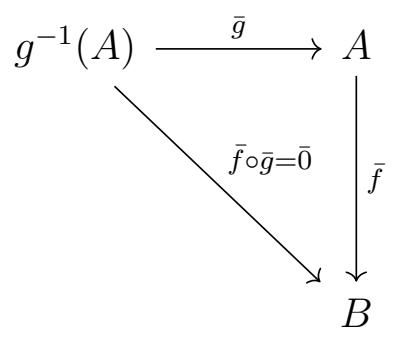

Therefore, kernel of $\bar{f}$ is defined as $g^{-1}(A)$ with the inclusion map $\bar{g}: g^{-1}(A) \rightarrow A$. Thus kernel of $\bar{f}$ is given as $\left(\left(\operatorname{Ker} f, g^{-1}(A)\right), \bar{g}\right)$, where the inclusion map is $g: \operatorname{Ker} f \rightarrow G$.

Similarly, cokernel of $\bar{f}$ is defined as $((H / \operatorname{Im} f, \pi(B)), \bar{\pi})$, where the projection map $\pi: H \rightarrow H / \operatorname{Imf}$ and $\bar{\pi}: B \rightarrow B_{H / \operatorname{Imf}}$.

Further, in order for an IF homomorphism $\bar{h}: C \rightarrow A$ of IFSG $C$ of $G$ to be normal (i.e., to be a kernel) $C$ must be equal to $g^{-1}(A)$. Hence for $G \neq\{\theta\}$, the IF homomorphism $\overline{1}: \chi_{\{\theta\}} \rightarrow \chi_{G}$ is a subobject of $\chi_{G}$ which is not a kernel and therefore $\mathbf{C}_{\mathbf{I F G}}$ is not an Abelian category. 


\section{Conclusion}

In this paper, we defined a function $\beta: \operatorname{Hom}(A, B) \rightarrow[0,1] \times[0,1]$ and showed that it is an intuitionistic fuzzy subgroup of $\operatorname{Hom}(A, B)$. Using this we proved that the cagegory $\mathbf{C}_{\mathbf{G}}$ of groups is a subcategory of the category $\mathbf{C}_{\mathbf{I F G}}$ of intuitionistic fuzzy groups. We also extablished a covariant functor from $\mathbf{C}_{\mathbf{G}}$ to $\mathbf{C}_{\mathbf{I F G}}$. Further, we proved that $\mathbf{C}_{\mathbf{I F G}}$ is a top category over $\mathbf{C}_{\mathbf{G}}$. Finally, we defined the concept of kernel and cokernel in $\mathbf{C}_{\mathbf{I F G}}$ and showed that $\mathbf{C}_{\mathbf{I F G}}$ is not an Abelian category. For further study we shall analyse the concept of inverse and direct systems in the category of intuitionistic fuzzy groups.

\section{References}

[1] Atanassov, K. T. (1986). Intuitionistic fuzzy sets. Fuzzy Sets and Systems, 20(1), 87-96.

[2] Atanassov, K. T. (1999). Intuitionistic Fuzzy Sets: Theory and Applications, Studies on Fuzziness and Soft Computing, 35, Heidelberg: Physica-Verlag.

[3] Awodey, S. (2005). Category Theory. Oxford University Press, 2 edition.

[4] Biswas, R. (1989). Intuitionistic fuzzy subgroup. Mathematical Forum, X, 37-46.

[5] Xu, C. (2008). New structure of Intuitionistic fuzzy groups. Huang, D. S., Wunsch, D. C., Levine. D. S., \& Jo, K. H. (eds) Advanced Intelligent Computing Theories and Applications. With Aspects of Contemporary Intelligent Computing Techniques. ICIC 2008. Communications in Computer and Information Science, vol 15. Springer, Berlin, Heidelberg, 145-152.

[6] Eilenberg, S., \& Mac Lane, S. (1945). General Theory of Natural Equivalences. American Mathematical Society, 58(2), 231-294.

[7] Fathi, M., \& Salleh A.R. (2009). Intuitionistic fuzzy groups. Asian Journal of Algebra, 2(1), $1-10$

[8] Kim, J., Lim, P. K., Lee, J. G., \& Hur, K. (2017). The category of intuitionistic fuzzy sets. Annals of Fuzzy Mathematics and Informatics, 14(6), 549-562.

[9] Leinster, T. (2014). Basic Category Theory. Cambridge Studies in Advanced Mathematics, Vol. 143.

[10] Sharma, P. K. (2011). Intuitionistic fuzzy groups. International Journal of Data warehousing and Mining, 1(1), 86-94.

[11] Sharma, P. K. (2012). On intuitionistic fuzzy Abelian subgroups, Advances in Fuzzy Sets and Systems, 12(1), 1-16. 
[12] Sharma, P. K. (2015). Intuitionistic Fuzzy Representations of Intuitionistic Fuzzy Groups, Asian Journal of Fuzzy and Applied Mathematics, 3(3), 81-94.

[13] Wyler, O. (1971). On the categories of general topology and topological algebra, Archiv der Mathematik, 22, 7-17.

[14] Yuan, X.-H., Li, H.-X., \& Stanley Lee, E. (2010). On the definition of the intuitionistic fuzzy subgroup, Computers \& Mathematics with Applications, 59, 3117-3129.

[15] Zadeh, L. A. (1965). Fuzzy Sets. Information and Control, 8, 338-353.

[16] Zhan, J., \& Tan, Z. (2004). Intuitionistic $M$-fuzzy groups. Soochow Journal of Mathematics, $30,85-90$. 